\title{
Photonic Crystal biosensor in spatial Fourier domain
}

\author{
Elewout Hallynck \\ Ghent University - imec \\ Photonics Research Group \\ Department of Information Technology (INTEC) \\ Sint-Pietersnieuwstraat 41 \\ 9000 Gent, Belgium \\ Email: elewout.hallynck@intec.ugent.be
}

\begin{abstract}
We propose a photonic crystal biosensor, operating at a single wavelength, based on analysis of resonant guided modes in the spatial Fourier domain. Sensitivities of 65 degrees per RIU and more have been simulated.
\end{abstract}

\section{INTRODUCTION}

Label-free biosensors today play (and will continue to do so) an important role in a broad range of applications (e.g. health care, military). Many sensing concepts have already been devised and published [1] but as will become clear in the following, there is still room for expansion.

In this paper, we propose a biosensor based on photonic crystals (PhCs). The most common photonic crystal based biosensors monitor the shift of a resonance in the frequency spectrum introduced either by a defect (i.e. point or waveguide) [2] or guided resonances [3]. The drawback of these concepts is that there is a need for a broadband light source. The structure we propose only requires a single wavelength source as we monitor a shift in the angular spectrum of a light beam coupled out by the PhC.

\section{SENSING IN THE SPATIAL FouRIER DOMAIN}

The working principle of the proposed photonic crystal $(\mathrm{PhC})$ biosensor is based on the coupling of true guided modes to resonant guided modes [4]. It can be seen that in a certain range of frequencies (depending on the configuration of the $\mathrm{PhC}$ ) light is coupled out as a Lorentzian beam under a certain angle (see Fig. 1) [5]. By changing the refractive index of the holes and cladding layers of the photonic crystal, the angle of this beam for a certain frequency will shift which allows us to use this structure as a sensor.

The simulations in this paper have been carried out using freely available FDTD based software [6] for a $0.3 a$ thick silicon PhC slab consisting of holes of $0.8 a$ diameter in a triangular lattice configuration (where $a$ is the lattice constant). A TE-polarised light pulse is coupled in in the $Г \mathrm{M}$ direction of the structure.

In order to obtain the power profile of the outcoupled light we use the electric and magnetic fields determined by the software to calculate the Poynting vector projected on the vertical direction. By applying a temporal Fourier transform followed by a spatial Fourier transform in two dimensions, we can study the angular spectrum of the Poynting vector for a

\author{
Peter Bienstman \\ Ghent University - imec \\ Photonics Research Group \\ Department of Information Technology (INTEC) \\ Sint-Pietersnieuwstraat 41 \\ 9000 Gent, Belgium \\ Email: peter.bienstman@intec.ugent.be
}

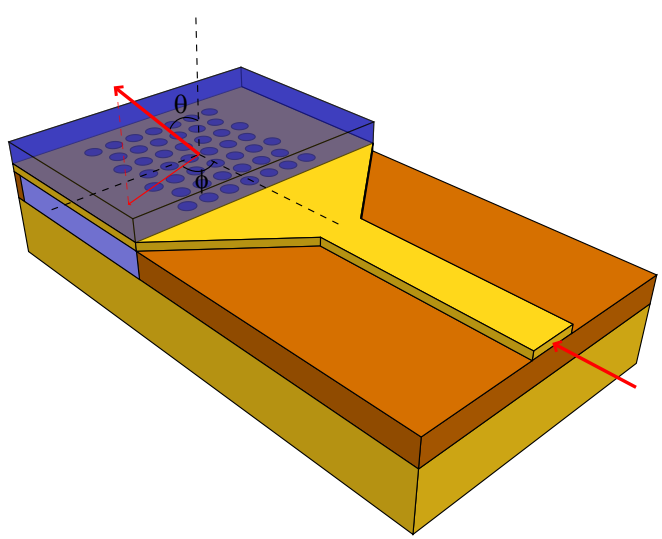

Fig. 1. Light is coupled out as a Lorentzian beam with inclination $\theta$ and azimuth $\phi$.

broad range of frequencies based on only one simulation. An example of the angular spectrum of the Poynting vector for a certain frequency can be seen in Fig. 2. We can verify that the position of the Lorentzian peak indeed shifts with varying refractive index of the $\mathrm{PhC}$.

\section{Simulation RESULTS}

When desiging a photonic crystal, several parameters can be tuned, each having an influence on the performance of the device. The most important parameter in this case is the type of cladding configuration of the $\mathrm{PhC}$ on which we will mainly focus. We will also shortly discuss a few other parameters and their effects.

\section{A. Influence of cladding configuration}

Depending on the method of analyte delivery and other fabrication considerations, there are three possible cladding configurations: the refractive index only changes in the $\mathrm{PhC}$ holes (the no cladding type), both the $\mathrm{PhC}$ holes and one of the cladding layers change while the other one remains air (asymmetric cladding) or both cladding layers and the $\mathrm{PhC}$ holes have their refractive index changed (symmetric cladding). For these three situations, several simulations have been carried, each time using a different refractive index so we can determine a shift per refractive index unit (RIU) of the 

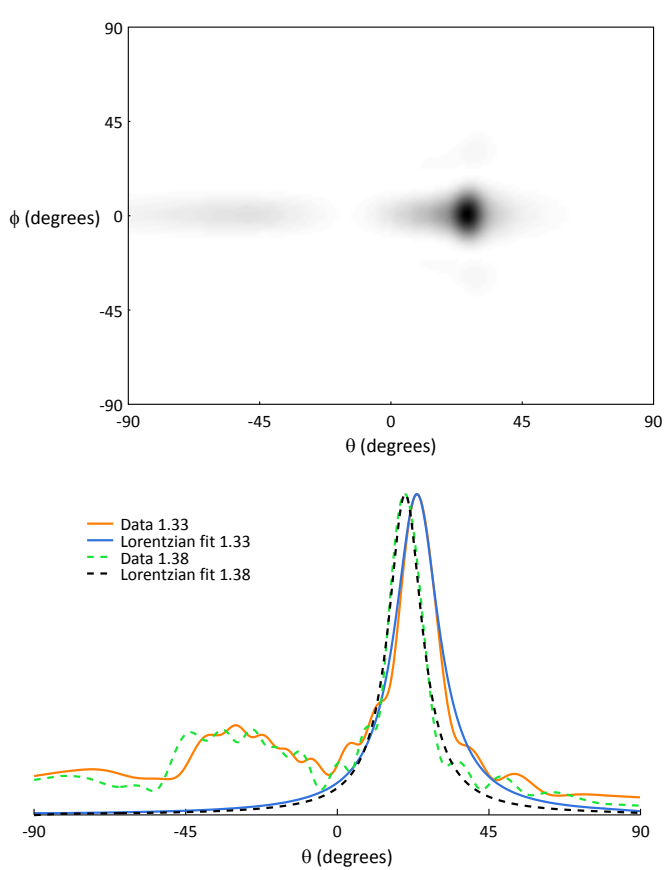

Fig. 2. Spectral image of outcoupled light (top) with cross-section at $\phi=0^{\circ}$ (bottom) where the Lorentzian shape can be distinguished. The refractive index of the cladding layers above and below the $\mathrm{PhC}$ as well as that of the holes all vary (symmetric cladding type). The normalised frequency is 0.53488

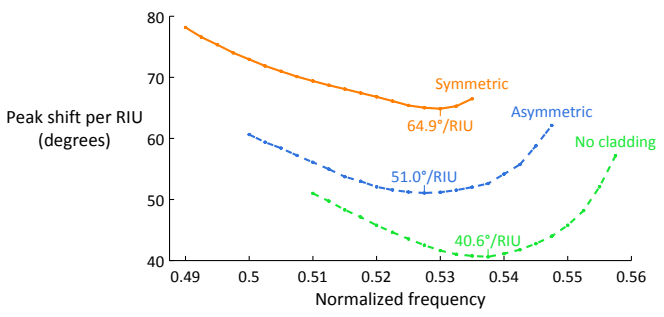

Fig. 3. Sensitivity for various cladding configurations. As expected, the symmetric cladding case is the most sensitive.

Lorentzian beam angle. The results of these simulations can be seen in Fig. 3.

As we would expect, the symmetric cladding configuration is the most sensitive with a minimal sensitivity of 65 degrees per RIU. It is recommended to operate the device at a high frequency because it can be shown that with increasing frequency, $\theta$ decreases (therefore making it easier to capture light in a measurement setup) and the width of the Lorentzian shape also decreases which in turn has a positive effect on the limit of detection.

\section{B. Other parameters}

Besides the different cladding configurations, we have also investigated the influence of several other parameters.

We changed the direction of light excitation from $Г M$ to $\Gamma \mathrm{K}$ and found that the Lorentzian becomes distorted and a portion of the light is also scattered at an angle $\phi=90^{\circ}$. As we are unable to properly fit a Lorentzian shape to these angular spectra we can make an estimation of the shift based on the peak maximum and find out that the sensitivity is roughly equal to that of the case where we excite light in the ГM direction.

As expected, the sensitivity increases with increasing hole diameter and slightly increases with decreasing slab thickness. The number of periods in the direction of light propagation has little influence on the beam width.

\section{CONCLUSION}

Based on the coupling of guided to free space modes, we have proposed a novel photonic crystal based biosensor. The major advantage of this device over resonance based sensors (e.g. Mach-Zehnder interferometers, ring resonators) is the ability to operate the device at a single wavelength. The structure, with a minimal sensitivity of 65 degrees per refractive index unit outperforms a surface plasmon resonance sensor in the angular regime [7]. Fabrication, compared to this SPR sensor, is also less complicated as there is no need for metal deposition or fabrication of a coupling prism.

\section{ACKNOWLEDGMENT}

The author would like to thank imec for providing a research grant. Simulations have been carried out using the Stevin Supercomputer Infrastructure at Ghent University.

\section{REFERENCES}

[1] X. Fan, I. M. White, S. I. Shopova, H. Zhu, J. D. Suter, and Y. Sun, "Sensitive optical biosensors for unlabeled targets: A review," Analytica Chimica Acta, vol. 620, no. 1-2, pp. 8 - 26, 2008 [Online]. Available: http://www.sciencedirect.com/science/article/B6TF4 4SJ2WV7-1/2/b631bf29724d89506db27aaac298026a

[2] E. Chow, A. Grot, L. W. Mirkarimi, M. Sigalas, and G. Girolami, "Ultracompact biochemical sensor built with two-dimensional photoniccrystal microcavity," Opt. Lett., vol. 29, no. 10, pp. 1093-1095, 2004. [Online]. Available: http://ol.osa.org/abstract.cfm?URI=ol-29-101093

[3] J. H. Schmid, W. Sinclair, J. García, S. Janz, J. Lapointe, D. Poitras, Y. Li, T. Mischki, G. Lopinski, P. Cheben, A. Delâge, A. Densmore, P. Waldron, and D.-X. Xu, "Silicon-on-insulator guided mode resonant grating for evanescent field molecular sensing," Opt. Express, vol. 17, no. 20, pp. 18371-18380, 2009. [Online]. Available: http://www.opticsexpress.org/abstract.cfm?URI=oe-17-20-18371

[4] S. Fan and J. D. Joannopoulos, "Analysis of guided resonances in photonic crystal slabs," Phys. Rev. B, vol. 65, no. 23, p. 235112, Jun 2002.

[5] E. Hallynck and P. Bienstman, "Photonic crystal biosensor based on angular spectrum analysis," Opt. Express, vol. 18 no. 17 , pp. 18 164-18 170, Aug 2010. [Online]. Available: http://www.opticsexpress.org/abstract.cfm?URI=oe-18-17-18164

[6] A. F. Oskooi, D. Roundy, M. Ibanescu, P. Bermel, J. D. Joannopoulos, and S. G. Johnson, "MEEP: A flexible free-software package for electromagnetic simulations by the FDTD method," Computer Physics Communications, vol. 181, pp. 687-702, January 2010.

[7] T. Kan, K. Matsumoto, and I. Shimoyama, "Nano-pillar structure for sensitivity enhancement of spr sensor," in Solid-State Sensors, Actuators and Microsystems Conference, 2009. TRANSDUCERS 2009. International, 21-25 2009, pp. 1481-1484. 